\section{New approaches for the treatment of diabetic macular oedema: recommendations by an expert panel}

F Bandello1, J Cunha-Vaz², NV Chong ${ }^{3}$, GE Lang ${ }^{4}$, P Massin ${ }^{5}$, P Mitchell ${ }^{6}$, M Porta ${ }^{7}$, C Prünte ${ }^{8,9}$, R Schlingemann ${ }^{10,11}$ and U Schmidt-Erfurth ${ }^{8}$

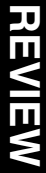

\begin{abstract}
The current standard therapy for patients with diabetic macular oedema (DME) - focal/grid laser photocoagulation - usually does not improve impaired vision, and many patients lose vision despite laser therapy. Recent approval of ranibizumab by the European Medicines Agency to treat visual impairment due to DME fulfils the previously unmet medical need for a treatment that can improve visual acuity (VA) in these patients. We reviewed 1- and 2-year clinical trial findings for ranibizumab used as treatment for DME to formulate evidence-based treatment recommendations in the context of this new therapy. DME with or without visual impairment should be considered for treatment when it fulfils the Early Treatment Diabetic Retinopathy Study (ETDRS) criteria for clinically significant oedema. For DME with centre involvement and associated vision loss due to DME, monthly ranibizumab monotherapy with treatment interruption and re-initiation based on VA stability is recommended. Laser therapy based on ETDRS guidelines is recommended for other forms of clinically significant DME without centre involvement or when no vision loss has occurred, despite centre involvement. Because these recommendations are based on randomised controlled trials of 1-2 years duration, guidance may need updating as long-term ranibizumab data become available and as additional therapeutic agents are assessed in clinical trials.
\end{abstract}

Eye (2012) 26, 485-493; doi:10.1038/eye.2011.337; published online 13 January 2012

Keywords: diabetic retinopathy; expert recommendations; vascular endothelial growth factor inhibitors

\section{Introduction}

Diabetic macular oedema (DME) is a leading cause of visual impairment in people with diabetes mellitus, ${ }^{1}$ and, if left untreated, $>50 \%$ of patients lose $>2$ lines of visual acuity (VA) within 2 years. ${ }^{2}$ DME mostly affects the working-age population, imposing a significant burden both on society and on individual patients ${ }^{3}$ - a burden that is expected to increase with the rising prevalence of diabetes. ${ }^{4-6}$

The standard therapy for visual impairment due to DME - focal and/or grid laser photocoagulation ${ }^{7,8}$ - is mostly only able to stabilise vision. In the Early Treatment Diabetic Retinopathy Study (ETDRS) criteria in patients with visual impairment due to DME, laser therapy reduced the relative risk of losing 15 letters of VA by $50 \%$ compared with deferring treatment. ${ }^{9}$ More recent trials reported gains of only 0.9 letters ${ }^{4}$ and three letters ${ }^{10}$ for patients receiving laser monotherapy according to ETDRS guidelines. Nevertheless, a small group of laser-treated patients $(21 \%)$ in the DRCR.net protocol I study did achieve a 15-letter improvement in VA at 2 years, suggesting a delayed benefit. ${ }^{11}$ Notwithstanding the importance of preventing further vision loss, there was until recently an unmet medical need for therapies that could restore VA in patients with DME who had visual impairment.

Although not fully elucidated, advances in understanding DME pathophysiology have launched the investigation of various pharmacological therapies, including those targeting vascular endothelial growth factor (VEGF), which is upregulated in eyes with $\mathrm{DME}_{,}^{12,13}$ and is a major mediator of increased retinal permeability. ${ }^{14}$ Investigational anti-VEGF therapies include aflibercept, pegaptanib, and bevacizumab (although bevacizumab is unlicensed for intraocular use). Although some
${ }^{1}$ Department of Ophthalmology, University Vita-Salute, Scientific Institute San Raffaele, Milano, Italy

${ }^{2}$ Association for Innovation and Biomedical Research on Light and Image, Coimbra, Portugal

${ }^{3}$ Oxford Eye Hospital, University of Oxford, Oxford, UK

${ }^{4}$ Department of Ophthalmology, University Eye Hospital, Ulm,

Germany

${ }^{5}$ Assistance Publique des Hôpitaux de Paris, Ophthalmology

Department, Hôpital Lariboisière, Paris, France

${ }^{6}$ Discipline of Ophthalmology, University of Sydney, Sydney, Australia

${ }^{7}$ Department of Internal Medicine, University of Turin, Turin, Italy

${ }^{8}$ Department of

Ophthalmology and Optometry, Medical University of

Vienna, Vienna, Austria

${ }^{9}$ Vista Klinik, Binningen, Switzerland

${ }^{10}$ Medical Retina Unit and Ocular Angiogenesis Group Department of Ophthalmology, University of Amsterdam, Academic Medical Centre, Amsterdam, The Netherlands

${ }^{11}$ Department of Clinical and Molecular Ophthalmogenetics, The Netherlands Institute for Neuroscience, Royal Netherlands Academy of Arts and Sciences (KNAW), Amsterdam,

The Netherlands

Correspondence: U Schmidt-Erfurth, Medical University of Vienna, Department of Ophthalmology and Optometry, Waehringer Guertel 18-20, Vienna 1090, Austria Tel: + 431404007941 ; Fax: + 431404007912 E-mail: ursula.schmidt-erfurth@ meduniwien.ac.at

Received: 22 August 2011 Accepted: 10 November 2011 Published online: 13 January 2012 
improvements in best-corrected VA (BCVA) has been observed with these agents, robust clinical trial evidence is currently limited. ${ }^{15-20}$

Ranibizumab (Lucentis Novartis Pharma AG, Basel, Switzerland and Genentech Inc., South San Francisco, CA, USA), a fully humanised monoclonal antibody fragment that binds to multiple variants of VEGF-A, was recently approved by the European Medicines Agency for the treatment of visual impairment due to $\mathrm{DME},{ }^{21}$ based on evidence from two pivotal trials, RESOLVE and RESTORE. ${ }^{4,5}$

New guidance that considers the availability of this agent and how it fits into the overall treatment algorithm for DME is therefore required. A panel of experts was convened, with sponsorship from Novartis Pharma AG, to review available treatment options for DME, including clinical trial evidence for ranibizumab in treating visual impairment due to this disease. The objective was to formulate treatment recommendations that provide the best possible VA outcomes while maintaining treatment regimens that are manageable and practical for both physicians and patients. This guidance is mostly based on 1-year data with ranibizumab, with the inclusion of 2-year data where available.

\section{Clinical trial evidence for ranibizumab in DME}

In this review, we focus on the most robust evidence for ranibizumab in patients with DME (prospective, randomised clinical trials with at least 6 months followup). To date, two phase II studies (RESOLVE ${ }^{5}$ and READ-2 ${ }^{22,23}$ ) and two phase III studies (RESTORE ${ }^{4}$ and DRCR.net protocol $\mathrm{I}^{10}$ ) have been completed using ranibizumab - a total of 1313 patients with DME (Table 1). The trial results discussed are data for the primary and secondary end points, which the clinical trials were sufficiently powered to investigate.

\section{Efficacy}

In READ-2, ranibizumab injections were administered at baseline and at months 1, 3, and 5, and focal/grid laser was administered at baseline and again at month 3 only if central subfield thickness (CST) was $\geq 250 \mu \mathrm{m}$. The mean change in BCVA from baseline to month 6 (primary end point) with ranibizumab monotherapy $(+7.24$ letters) was superior to laser monotherapy ( -0.43 letters, $P=0.0001)$; however, the BCVA change with combination therapy was not significantly different from that of either ranibizumab or laser monotherapy at this timepoint ( +3.8 letters; $P=0.08$ for both comparisons). ${ }^{22}$ In total, 101 patients completed 24 months follow-up; the mean BCVA improvement at month 6 with ranibizumab monotherapy was sustained to month 24 and these improvements were numerically higher than with combination therapy $(+7.7$ vs +6.8 letters, respectively) (Figure 1a). ${ }^{23}$ For the 74 patients who completed 36 months follow-up, mean BCVA continued to improve with mean gains of +10.3 letters with ranibizumab monotherapy and +9.5 letters with combination therapy vs +1.4 letters for patients initially randomised to laser monotherapy (Table 2). ${ }^{24}$ Interestingly, adding laser to ranibizumab resulted in fewer ranibizumab injections without a major disadvantage in visual outcome at 2 and 3 years (Table 2). ${ }^{23,24}$

In RESOLVE, ranibizumab was administered as three consecutive monthly ranibizumab $(0.3$ or $0.5 \mathrm{mg}$ ) injections, followed by an as-needed regimen with predefined retreatment criteria. Dose doubling and rescue laser were permitted at investigator discretion. The primary efficacy outcome was the mean average change in BCVA over 12 months, defined as the difference between BCVA at baseline and the average of BCVA values measured at months $1-12$. This is considered to be a more stringent regulatory end point than mean change in BCVA as it incorporates the treatment effect over the entire treatment period. The mean average change in BCVA with ranibizumab treatment was superior to that of the sham control group (Table 1). At 12 months, ranibizumab-treated patients gained 10.3 letters in BCVA, with a mean of 10 injections vs a loss of 1.4 letters in the sham group $(P<0.0001)$ (Figure 1b). More patients receiving ranibizumab gained $\geq 15$ letters compared with patients receiving sham injections (Table 1 ); $2.9 \%$ of the ranibizumab group and $20.4 \%$ of the sham group lost $\geq 15$ letters. Dose doubling was more frequent in the sham arm $(91.8 \%)$ than in the ranibizumab arm $(68.6 \%)$, whereas a higher proportion of patients in the sham arm received rescue laser therapy (32.7\%) compared with the ranibizumab $\operatorname{arm}(4.9 \%){ }^{5}$

RESTORE was a 12-month, randomised, phase III study in which 345 patients with visual impairment due to DME received ranibizumab monotherapy, and ranibizumab combined with laser or laser monotherapy. Ranibizumab injections were administered monthly (for a minimum of three injections) until disease stability was achieved and were subsequently re-initiated when there was evidence of disease activity with predefined cessation and re-initiation criteria. Laser was administered in accordance with ETDRS guidelines. ${ }^{25}$ The primary end point for RESTORE was also the mean average change in BCVA, which demonstrated the superiority of ranibizumab, either as monotherapy or when combined with laser, compared with laser monotherapy (Table 1). Mean BCVA change at month 12 was also significantly better for ranibizumab-treated patients than for patients receiving laser monotherapy 
Table 1 Summary of outcomes from prospective RCTs with ranibizumab in diabetic macular oedema

\begin{tabular}{|c|c|c|c|c|}
\hline & $R E A D-2^{23}(\mathrm{~N}=126)$ & $\operatorname{RESOLVE}^{5}(\mathrm{~N}=151)$ & RESTORE $^{4}(\mathrm{~N}=345)$ & DRCR.net protocol I $I^{11}(\mathrm{~N}=691)$ \\
\hline $\begin{array}{l}\text { Study design and } \\
\text { duration }\end{array}$ & Phase II RCT, 36 months $^{\mathrm{a}}$ & $\begin{array}{l}\text { Phase II RCT, } 12 \\
\text { months }\end{array}$ & Phase III RCT, 12 months & Phase III RCT, 60 months $^{\text {b }}$ \\
\hline Study treatment & $\begin{array}{l}\text { RBZ } 0.5 \mathrm{mg}(n=42) \\
\text { RBZ } 0.5 \mathrm{mg}+\text { laser }(n=42)\end{array}$ & $\begin{array}{l}\text { RBZ 0.3/0.6 mg PRN } \\
(n=51) \\
\text { RBZ 0.5/1.0 mg PRN } \\
(n=51)\end{array}$ & $\begin{array}{l}\text { RBZ } 0.5 \mathrm{mg} \text { PRN }(n=116) \\
\text { RBZ } 0.5 \mathrm{mg} \text { PRN + laser }(n=118)\end{array}$ & $\begin{array}{l}\text { RBZ 0.5 mg PRN + prompt laser } \\
(n=187)^{\mathrm{c}} \\
\text { RBZ 0.5 mg PRN + deferred laser } \\
(n=188)^{\mathrm{c}}\end{array}$ \\
\hline Comparator & $\begin{array}{l}\text { Laser months } 0-6 \text {; eligible } \\
\text { ranibizumab after month } \\
6(n=42)\end{array}$ & $\begin{array}{l}\text { Sham injection PRN } \\
(n=49)\end{array}$ & Laser PRN $(n=111)$ & $\begin{array}{l}\text { Triamcinolone } 4 \mathrm{mg} \\
\text { PRN + prompt laser }(n=186)^{\mathrm{c}} \\
\text { Sham injection PRN }+ \text { prompt } \\
\text { laser }(n=293)^{c}\end{array}$ \\
\hline $\begin{array}{l}\text { Mean number of } \\
\text { ranibizumab } \\
\text { injections }\end{array}$ & $\begin{array}{l}\text { RBZ } 0.5 \mathrm{mg}: 4 \\
\text { RBZ } 0.5 \mathrm{mg} \text { + laser: } 2\end{array}$ & RBZ pooled: 10.2 & $\begin{array}{l}\text { RBZ } 0.5 \mathrm{mg}: 7.0 \\
\text { RBZ } 0.5 \mathrm{mg} \text { + laser: } 6.8\end{array}$ & $\begin{array}{l}\text { RBZ } 0.5 \mathrm{mg} \text { + prompt laser: } 8^{\mathrm{d}} \\
\text { RBZ } 0.5 \mathrm{mg} \text { + deferred laser: } 9^{\mathrm{d}}\end{array}$ \\
\hline $\begin{array}{l}\text { BCVA change } \\
\text { from baseline }\end{array}$ & $\begin{array}{l}\text { Month 6: } \\
\text { RBZ 0.5 mg: }+7.24 \\
(P=0.0001) \\
\text { RBZ 0.5 mg + laser: } \\
+3.8(P=0.08) \\
\text { Laser: }-0.43\end{array}$ & $\begin{array}{l}\text { Month 12: } \\
\text { RBZ pooled: }+10.3 \\
(P<0.0001) \\
\text { Sham: }-1.4\end{array}$ & $\begin{array}{l}\text { Month 12: RBZ } 0.5 \mathrm{mg}:+6.8 \\
(P<0.0001) \\
\text { RBZ } 0.5 \mathrm{mg}+\text { laser: }+6.4 \\
(P=0.0004) \\
\text { Laser: }+0.9\end{array}$ & $\begin{array}{l}\text { Month } 12 \text { : } \\
\text { RBZ } 0.5 \mathrm{mg}+\text { prompt } \\
\text { laser: }+9(P<0.001) \\
\text { RBZ } 0.5 \mathrm{mg}+\text { deferred } \\
\text { laser: }+9(P<0.001) \\
\text { Triamcinolone + prompt } \\
\text { laser: }+4(P=0.31) \\
\text { Sham + prompt laser: }+3\end{array}$ \\
\hline $\begin{array}{l}\text { BCVA mean } \\
\text { average change } \\
\text { from baseline to } \\
\text { month } 12\end{array}$ & NR & $\begin{array}{l}\text { RBZ pooled: } 7.8 \\
(P<0.0001) \\
\text { Sham: }-0.1\end{array}$ & $\begin{array}{l}\text { RBZ } 0.5 \mathrm{mg}:+6.1(P<0.0001) \\
\text { RBZ } 0.5 \mathrm{mg} \text { + laser: }+5.9 \\
(P<0.0001) \\
\text { Laser: }+0.8\end{array}$ & NR \\
\hline Patients gaining & RBZ $0.5 \mathrm{mg}: 22$ & RBZ pooled: 32.4 & RBZ 0.5 mg: 22.6 & RBZ $0.5 \mathrm{mg}+$ prompt laser: 30 \\
\hline 15 letters (\%) & $\begin{array}{l}\text { RBZ } 0.5 \mathrm{mg} \text { + laser: } 8 \\
\text { Laser: } 0\end{array}$ & Sham: 10.2 & $\begin{array}{l}\text { RBZ } 0.5 \mathrm{mg} \text { + laser: } 22.9 \\
\text { Laser: } 8.2\end{array}$ & $\begin{array}{l}\text { RBZ } 0.5 \mathrm{mg} \text { + deferred laser: } 28 \\
\text { Triamcinolone + prompt laser: } 21 \\
\text { Sham + prompt laser: } 15\end{array}$ \\
\hline $\begin{array}{l}\text { CRT change from } \\
\text { baseline }\end{array}$ & $\begin{array}{l}\text { Month } 6 \text { : RBZ } 0.5 \mathrm{mg}: \\
-106.3 \mu \mathrm{m}^{\mathrm{b}}(P<0.0001) \\
\text { RBZ } 0.5 \mathrm{mg}+\text { laser: } \\
-117.2 \mu \mathrm{m}^{\mathrm{b}}(P<0.0001) \\
\text { Laser only: }-82.2 \mu \mathrm{m}^{\mathrm{b}} \\
(P=0.003)\end{array}$ & $\begin{array}{l}\text { Month 12: RBZ pooled: } \\
-194.2 \mu \mathrm{m}(P<0.0001) \\
\text { Sham: }-48.4 \mu \mathrm{m}\end{array}$ & $\begin{array}{l}\text { Month 12: RBZ } 0.5 \mathrm{mg}:-118.7 \mu \mathrm{m} \\
(P=0.0002) \\
\text { RBZ } 0.5 \mathrm{mg} \text { + laser: }-128.3 \mu \mathrm{m} \\
(P<0.0001) \\
\text { Laser: }-61.3 \mu \mathrm{m}\end{array}$ & $\begin{array}{l}\text { Month 12: RBZ } 0.5 \mathrm{mg}+\text { prompt } \\
\text { laser: }-131 \mu \mathrm{m}(P<0.001) \\
\text { RBZ } 0.5 \mathrm{mg}+\text { deferred } \\
\text { laser: }-137 \mu \mathrm{m}(P<0.001) \\
\text { Triamcinolone }+ \text { prompt } \\
\text { laser: }-127 \mu \mathrm{m}(P<0.001) \\
\text { Sham + prompt laser: }-102 \mu \mathrm{m}\end{array}$ \\
\hline
\end{tabular}

Abbreviations: BCVA, best-corrected visual acuity; CRT, central retinal thickness; NR, not reported; PRN, as needed; RBZ, ranibizumab; RCT, randomised controlled trial.

$P$-values are $v s$ sham or laser controls.

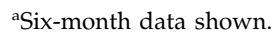

${ }^{\text {b}}$ Twelve-month data shown.

'Number of eyes included in the primary end point analyses at month 12 .

${ }^{\mathrm{d}}$ Median value shown.

$(+6.8$ letters with ranibizumab monotherapy $(P<0.0001)$

and +6.4 letters with combination therapy $(P=0.0004)$

vs +0.9 letters with laser monotherapy; Figure $1 c)$.

Similarly, more patients treated with ranibizumab experienced an improvement of $\geq 10$ letters than those on laser monotherapy: $37.4 \%$ with ranibizumab monotherapy $(P<0.0001)$ and $43.2 \%$ with combination therapy $(P<0.0001)$ vs $15.5 \%$ with laser monotherapy. Only one patient $(0.9 \%)$ receiving ranibizumab monotherapy experienced a loss of $\geq 15$ letters at month 12 vs four patients (3.4\%) in the combination therapy group and nine patients $(8.2 \%)$ in the laser monotherapy group. ${ }^{4}$

The DRCR.net protocol I study was an independent, randomised, phase III trial in 854 eyes of 691 patients with centre-involved retinal thickening due to DME.
Patients received ranibizumab plus either prompt (within 3-10 days of ranibizumab injection) or deferred ( $\geq 24$ weeks after injection) laser, triamcinolone plus laser or laser monotherapy. Ranibizumab was administered at baseline every 4 weeks to week 12 . From week 16, retreatment was guided by predefined VA and optical coherence tomography (OCT) criteria, but was at investigator discretion. The study has a 5-year follow-up period; to date, data for $1-^{10}$ and 2-year outcomes ${ }^{11}$ are reported. Mean BCVA change from baseline to year 1 was significantly better with ranibizumab in combination with either prompt or deferred laser therapy than with laser monotherapy (Table 1). ${ }^{10}$ The mean improvement of nine letters was achieved with a median of eight and nine ranibizumab injections in the prompt and deferred laser groups, respectively. In the first year, the ranibizumab 

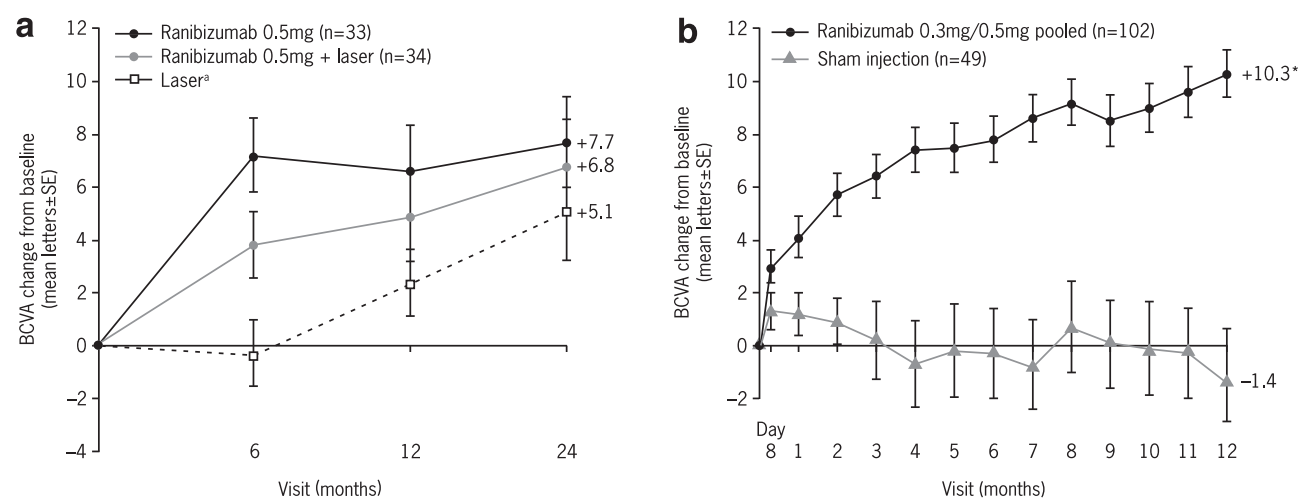
C $\rightarrow$ Ranibizumab $0.5 \mathrm{mg}(\mathrm{n}=115)$ $\rightarrow$ Ranibizumab $0.5 \mathrm{mg}+\operatorname{laser}(n=118)$ $\rightarrow-$ Laser $(n=110)$
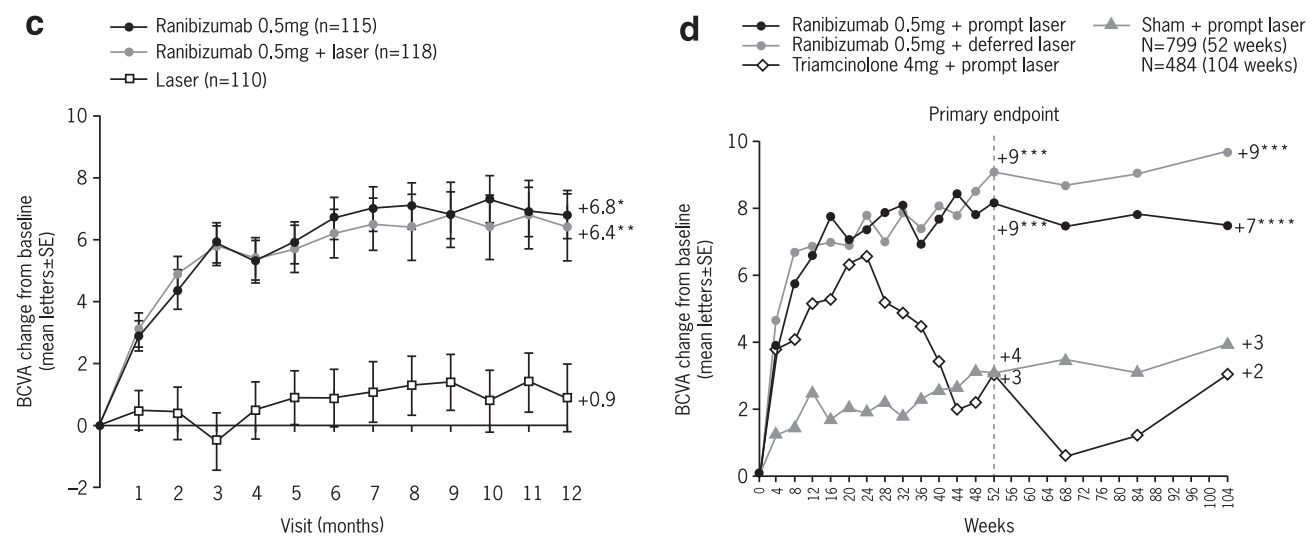

Figure 1 BCVA outcomes over time in prospective, randomised clinical trials with ranibizumab in DME. (a) READ-2; (b) RESOLVE;

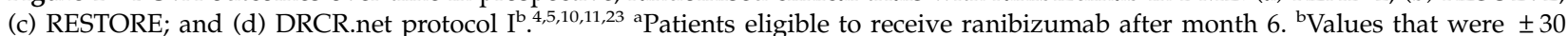
letters were assigned a value of $30 .{ }^{*} P<0.0001$ vs control; ${ }^{* *} P=0.0004$ vs control; ${ }^{* * *} P<0.001$ vs sham + prompt laser; ${ }^{* * * *} P=0.03$ vs sham + prompt laser. BCVA, best-corrected visual acuity; DME, diabetic macular oedema.

Table 2 BCVA outcomes and ranibuizumab injection frequency in the ranibizumab treatment arms of READ-2 and DRCR.net protocol I

\begin{tabular}{|c|c|c|c|c|c|}
\hline & \multicolumn{3}{|c|}{$R E A D-2^{22-24}$} & \multicolumn{2}{|c|}{ DRCR.net protocol $I^{10,11}$} \\
\hline & 6 months $(\mathrm{N}=126)$ & 24 months $(\mathrm{N}=101)$ & 36 months $(\mathrm{N}=74)$ & 12 months $(\mathrm{N}=691)$ & 24 months $(\mathrm{N}=526)$ \\
\hline Mean BCVA & $\begin{array}{l}\text { RBZ } 0.5 \mathrm{mg}:+7.24 \\
\text { RBZ } 0.5 \mathrm{mg}+ \\
\text { laser: }+3.8\end{array}$ & $\begin{array}{l}\text { RBZ } 0.5 \mathrm{mg}:+7.7 \\
\text { RBZ } 0.5 \mathrm{mg}+ \\
\text { laser: }+6.8\end{array}$ & $\begin{array}{l}\text { RBZ } 0.5 \mathrm{mg}:+10.3 \\
\text { RBZ } 0.5 \mathrm{mg}+ \\
\text { laser: }+9.5\end{array}$ & $\begin{array}{l}\text { RBZ } 0.5 \mathrm{mg}+\text { prompt } \\
\text { laser: }+9 \\
\text { RBZ } 0.5 \mathrm{mg}+\text { deferred } \\
\text { laser: }+9\end{array}$ & $\begin{array}{l}\text { RBZ } \\
0.5 \mathrm{mg}+\text { prompt } \\
\text { laser: }+7 \\
\text { RBZ } \\
0.5 \mathrm{mg}+\text { deferred } \\
\text { laser: }+9\end{array}$ \\
\hline $\begin{array}{l}\text { Mean number of } \\
\text { ranibizumab } \\
\text { injections }\end{array}$ & $\begin{array}{l}\text { RBZ } 0.5 \mathrm{mg}: 4 \\
\text { RBZ } 0.5 \mathrm{mg}+ \\
\text { laser: } 2\end{array}$ & $\begin{array}{l}\text { Months 6-24: } \\
\text { RBZ 0.5 mg: } 5.3 \\
\text { RBZ } 0.5 \mathrm{mg}+ \\
\text { laser: } 2.9\end{array}$ & $\begin{array}{l}\text { Months 24-36: } \\
\text { RBZ 0.5 mg: } 5.5 \\
\text { RBZ } 0.5 \mathrm{mg}+ \\
\text { laser: } 2.9\end{array}$ & $\begin{array}{l}\text { RBZ } 0.5 \mathrm{mg}+\text { prompt } \\
\text { laser: } 8^{\mathrm{a}} \\
\text { RBZ } 0.5 \mathrm{mg}+\text { deferred } \\
\text { laser: } 9^{\mathrm{a}}\end{array}$ & $\begin{array}{l}\text { Months 12-24: RBZ } \\
0.5 \mathrm{mg}+\text { prompt } \\
\text { laser: } 2^{\mathrm{a}} \\
\text { RBZ } \\
0.5 \mathrm{mg}+\text { deferred } \\
\text { laser: } 3^{\mathrm{a}}\end{array}$ \\
\hline
\end{tabular}

Abbreviation: BCVA, best-corrected visual acuity.

${ }^{\mathrm{a}}$ Median value shown.

plus prompt laser group received a median of two laser treatments, whereas $72 \%$ of patients receiving ranibizumab plus deferred laser did not receive laser treatment. BCVA improvements at year 1 were sustained during year 2, with a median of only two and three injections in the ranibizumab plus prompt and deferred laser groups, respectively (Figure 1d and Table 2). ${ }^{10,11}$ 
Subgroup analyses of data from the RESOLVE, RESTORE, and DRCR.net trials have been carried out according to patient baseline characteristics, including previous treatment, type of DME (focal or diffuse), baseline central retinal thickness (CRT), or baseline BCVA.

The mean average change in BCVA was consistently higher with ranibizumab in each subgroup of RESOLVE, with similar treatment differences between ranibizumab and sham controls. Similarly, the RESTORE study demonstrated the superiority of ranibizumab (either monotherapy or adjunctive to laser) to laser monotherapy in each subgroup (Figure 2). Interestingly, although the RESTORE findings indicated that patients with VA $>73$ letters had lower VA gains with ranibizumab than did patients in worse VA categories, this group with better baseline VA also had the greatest potential to experience a mean loss of VA when treated with laser monotherapy. Patients with CRT $<300 \mu \mathrm{m}$ also had lower VA gains with ranibizumab than did those with thicker baseline CRT values. These findings correlate well with the DRCR.net protocol I study in which there was no obvious clinically important difference in the 1-year results based on previous treatment for DME, baseline VA or CST, baseline level of diabetic retinopathy, or type of DME; however, patients with poorer VA ( $\leq 65$ letters) or greater CST ( $\geq 400 \mu \mathrm{m})$ experienced a greater VA benefit in all treatment groups than did patients with VA $\geq 66$ letters or CST $<400 \mu \mathrm{m}$. ${ }^{10}$

Although studies were not powered for these specific subgroup analyses, they suggest that baseline VA and CRT may influence VA treatment outcomes with ranibizumab; however, a treatment benefit was observed over controls across all subcategories. ${ }^{4,10,26}$

\section{Safety}

Patients with diabetes face various potential systemic complications, in addition to eye disease, particularly an increased risk for cardiovascular events. ${ }^{27}$ The safety and

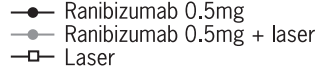

a

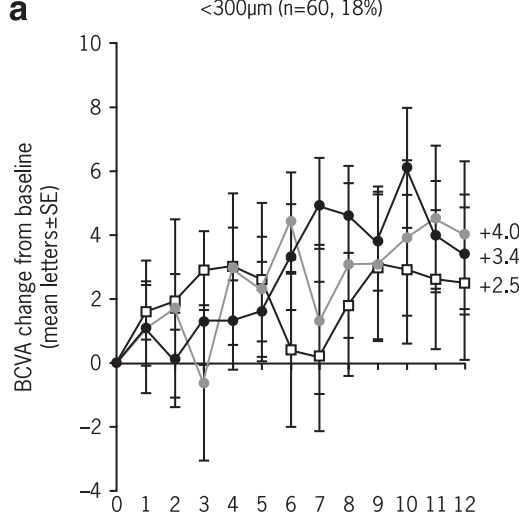

Visit (months)

b

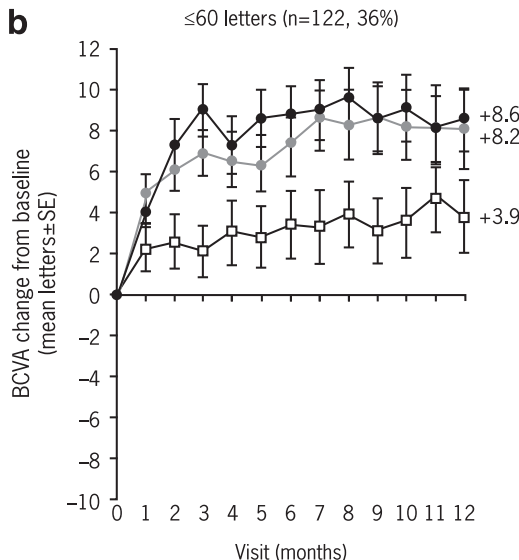

$300-400 \mu m(n=103,31 \%)$

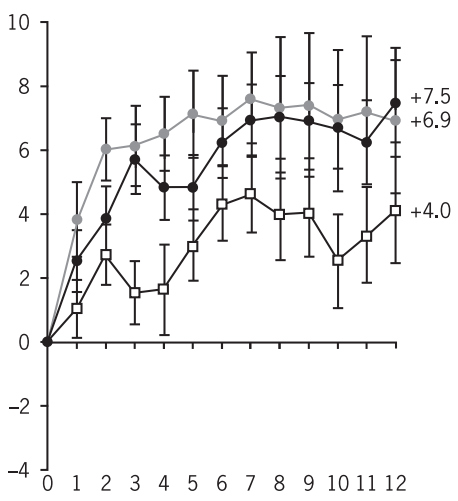

Visit (months)

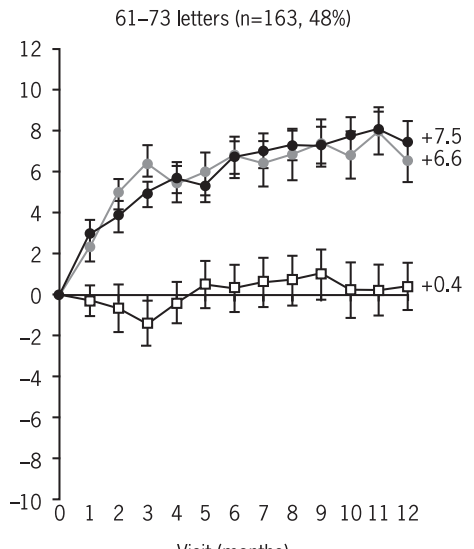

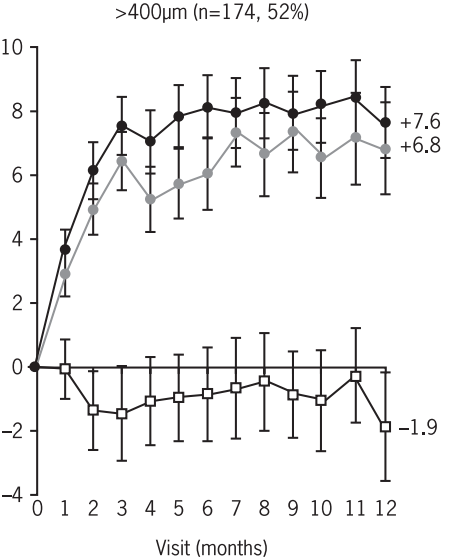

$>73$ letters $(n=58,17 \%)$

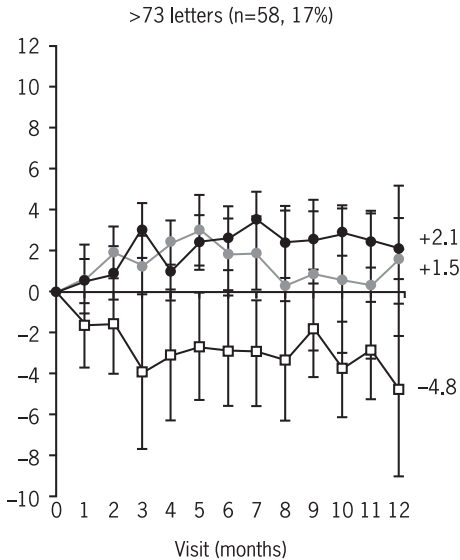

Figure 2 RESTORE subgroup analysis based on (a) baseline CRT and (b) baseline VA. ${ }^{4}$ CRT, central retinal thickness; DME, diabetic macular oedema; SE, standard error; VA, visual acuity. 
tolerability of new therapies in DME is therefore of critical importance.

Clinical studies have consistently demonstrated a favourable safety and tolerability profile with ranibizumab in patients with DME, with no imbalances in the rates of serious adverse events (SAEs) between ranibizumab treatment and control arms. ${ }^{4,5,10,23}$ Ocular SAEs with ranibizumab monotherapy were reported in four $(3.9 \%)$ patients over 1 year in RESOLVE, with none reported in RESTORE (at 1 year) or READ-2 (at 6 months). Ocular SAEs with ranibizumab combined with laser were reported in two (1.7\%) patients in RESTORE. There was a low incidence of endophthalmitis in ranibizumab treatment arms, with no cases in READ-2 or RESTORE, two cases in RESOLVE, and two cases during 1 year of treatment in DRCR.net protocol I (one in each of the ranibizumab arms).

In both RESOLVE and RESTORE, non-ocular SAEs were well balanced between ranibizumab and control groups. The incidence of non-ocular SAEs was 13.7 and $12.2 \%$ in the ranibizumab monotherapy and sham groups of RESOLVE, respectively, and 12.2, 5.8, and $10 \%$ in the ranibizumab monotherapy, combination, and laser monotherapy arms in RESTORE, respectively. There was no indication of an increased rate of cardiovascular or cerebrovascular events in ranibizumab groups $v s$ controls. ${ }^{4,5,10,23}$

\section{Recommendations for the treatment of DME}

The goal of treatment with laser photocoagulation was mostly VA stabilisation. With the approval of ranibizumab for the treatment of visual impairment due to DME, the goal of therapy should now primarily be the improvement or restoration of VA, with stabilisation of vision and prevention of further vision loss as a key secondary goal.

Treatment recommendations for DME are based on involvement of the centre of the macula (Figure 3). No new recommendations for the treatment of DME without centre involvement, or for DME with centre involvement without vision loss, are required as current ETDRS guidelines remain appropriate. ${ }^{25}$ Ranibizumab monotherapy is recommended for the treatment of DME with centre involvement, with vision loss considered due to DME. In that respect, it is important to

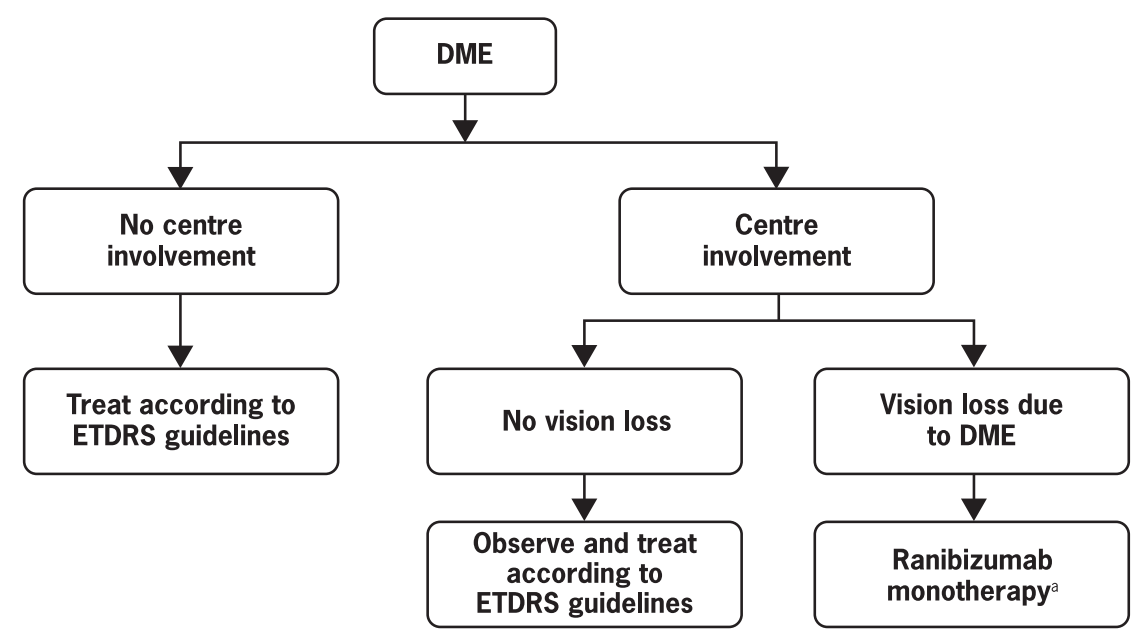

Summary of ETDRS guidelines

1) Focal photocoagulation treatment of individual microaneurysms that fill with fluorescein and/or leak, as well as other points of leakage such as intraretinal microvascular abnormalities or short capillary segments.

Optional: Treatment of microaneurysms (or punctate haemorrhages) $<125 \mu \mathrm{m}$ in longest diameter that did not fill with fluorescein; leaks within haemorrhages; treatment of microaneurysms or other focal points of leakage in the retina further than 2 DD from the centre of the macula.

Avoid: Treatment of nerve fibre layer retinal haemorrhage (flame or splinter haemorrhage) and blot haemorrhage $>125 \mu \mathrm{m}$ in size.

2) Grid laser treatment of areas of thickened retina showing diffuse fluorescein leakage and/or capillary dropout.

Figure 3 Treatment algorithm for DME. ${ }^{a}$ Data at 1 year suggest that concomitant administration of ranibizumab and laser is not harmful; however, no added benefit of adding laser to ranibizumab therapy has been demonstrated. The addition of laser can be considered at the physician's discretion. When given on the same day, ranibizumab should be administered at least 30 min after laser photocoagulation. DD, disk diameter; DME, diabetic macular oedema; ETDRS, Early Treatment Diabetic Retinopathy Study. 
exclude other potential causes of vision loss, such as epiretinal membrane, vitreomacular traction, or macular ischaemia, and other conditions such as cataract or glaucoma.

Systemic factors are key to the management of diabetes, and patients should attempt to achieve optimal control of haemoglobin A1c, lipid levels, and especially blood pressure. ${ }^{28}$ Intensive control of blood glucose and hypertension reduces development of clinically significant macular oedema. ${ }^{29,30}$ Achieving control of systemic factors reduces retinal thickness and improves VA to some extent in patients with very mild DME in the absence of other interventions. ${ }^{31}$

Treatment with thiazolidinediones, a class of antihyperglycaemic agents known to cause fluid retention, may be associated with $\mathrm{DME}^{32}$ and their discontinuation was reported to result in DME regression. ${ }^{33}$ Although this association is unconfirmed, ${ }^{34}$ discontinuation of thiazolidinediones in patients with DME is currently recommended. Surgery should be considered in patients with DME owing to a significant epiretinal membrane or demonstrated vitreomacular traction.

Proliferative diabetic retinopathy does not fall under the scope of these recommendations; however, caution should be exercised with the use of anti-VEGF agents in patients who have DME with co-existing proliferative diabetic retinopathy to avoid accelerated fibrosis and scarring due to the angio-fibrotic switch. ${ }^{35}$ Such damage can lead to rapid formation of tractional retinal detachment. ${ }^{35,36}$

\section{Guidance for ranibizumab therapy}

\section{Monotherapy vs combination therapy}

Initiating ranibizumab monotherapy is the preferred approach for visual impairment due to DME. As yet, the role of adjunctive laser is unclear. In RESTORE, laser in combination with ranibizumab did not improve outcomes compared with ranibizumab monotherapy; thus, the principal rationale for adjunctive laser is to reduce the number of ranibizumab injections. Two-year outcomes from the READ-2 and DRCR.net studies provided the first evidence for a reduction in the number of ranibizumab injections when combined with laser. ${ }^{10,23}$ As VA improvements with laser occur very slowly, ${ }^{23}$ long-term data are required to confirm whether adding laser to ranibizumab confers any additional benefit in terms of VA and patient quality of life, and thus clinical judgement should prevail.

\section{Treatment frequency and monitoring}

The approved posology for ranibizumab and 1-year outcomes in clinical trials were considered in our recommendations: ranibizumab injections should be initiated on a monthly basis, with monthly VA monitoring, until VA stability is achieved for a period of at least two consecutive visits or until normal VA is achieved. Treatment with ranibizumab can be interrupted when VA stability is achieved. This is defined in the approved posology as no further documented VA improvement over three consecutive visits while on treatment. However, monitoring should be continued on a monthly basis and, if VA deteriorates owing to DME, monthly injections should be re-initiated until stability is again achieved.

In clinical practice, decisions on treatment continuation, interruption, and re-initiation are most likely to be based on the combination of OCT and VA. OCT is a useful additional tool for monitoring DME stability and for confirming whether vision loss is due to DME. Fluorescein angiography assessment may be needed in specific situations (eg, development of unexplained visual loss).

On the basis of the above treatment criteria, the frequency of injections in the first year of treatment with ranibizumab can be expected to be similar to that in the RESTORE trial (ie, seven injections (five in the first 6 months and two in the second 6 months)). ${ }^{4}$ There may be an opportunity to reduce the frequency of monitoring in the second year of treatment, but no recommendation can yet be made based on the currently available evidence.

Patients with progressive worsening of VA over three consecutive visits in the presence of active DME (ie, when other causes of vision loss have been excluded) can be considered as non-responders to ranibizumab therapy. In these patients, it is recommended to discontinue ranibizumab and consider other treatment options, in particular laser treatment or intravitreal steroids.

\section{Special circumstances}

As DME is a complication of an underlying systemic condition, the rate of bilateral cases of visual impairment due to DME is likely to be higher than with other ocular diseases, such as age-related macular degeneration, which may substantially increase the treatment burden in patients with DME. Currently, there are limited data to guide the bilateral treatment of DME. In patients with central involvement and vision loss due to DME, physicians should consider treating the second eye either with ranibizumab in a manner similar to the first eye or with a combination of ranibizumab and laser treatment to reduce the number of intravitreal injections.

Ranibizumab has provided similar benefits in both pseudophakic and phakic eyes, ${ }^{10}$ however, further studies are required to determine whether presurgical 
administration of ranibizumab is beneficial in patients with DME undergoing cataract surgery.

Patients with a history of previous stroke were excluded from RESTORE, but not from the RESOLVE and DRCR.net protocol I studies. Although no signal of increased risk of arterial thromboembolic events was observed, caution needs to be exercised in the use of ranibizumab in patients with DME.

The use of ranibizumab in pregnancy has not been investigated. Therefore, ranibizumab should not be used during pregnancy, unless the expected benefit outweighs the potential risk to the foetus. Women of childbearing potential should have pregnancy tests before treatment is administered and should use effective contraception during treatment.

\section{Future considerations}

The recommendations for treating visual impairment due to DME with ranibizumab are expected to be updated as more long-term data become available, particularly with regard to the second year of treatment. The RESTORE extension study is ongoing, which will provide data for up to 3 years of treatment with ranibizumab. Likewise, the DRCR.net protocol I has been extended for up to 5 years. The REVEAL, RETAIN, RISE, and RIDE studies are also ongoing, providing additional data for 2-3 years of treatment. Other newly approved therapies will also need to be considered.

Key unanswered questions currently include the role of laser combination therapy in the treatment of visual impairment due to DME and precise recommendations around bilateral treatment with ranibizumab. In addition, there remains an unmet medical need for therapies that improve vision in patients who do not respond to ranibizumab, as between 3 and $5 \%$ of ranibizumab-treated patients lost $\geq 10$ BCVA letters over 12 months. ${ }^{4,5,10}$

Endeavours towards reducing the treatment burden in patients with DME are desirable. The current aim of reducing the frequency of monitoring required in the second year of treatment may go some way to alleviating the burden. Other steps may also include the establishment of remote self-monitoring for patients, which could help to provide an alternative option to frequent clinic visits in the future.

\section{Conflict of interest}

FB is an advisory board member for Alcon, Allergan, Bausch \& Lomb, Bayer, Novartis Pharma AG, Pfizer, and Thea. JC-V is a consultant for Novartis, Pfizer, Alcon, Bayer, Allergan, and Astellas Pharma Europe. NVC is a consultant for Novartis Pharma AG, Pfizer, Allergan, Merck, Bayer, and Iridex. GEL received consultancy fees and travel reimbursement from Novartis Pharma AG. PMa received consultancy fees from Novartis Pharma AG, Allergan, Fovea Pharmaceutical, Sanofi Aventis, and Takeda and has also been paid lecture fees by Novartis Pharma AG, Allergan, and Takeda. PMi received consultancy and lecture fees and travel reimbursements from Novartis Pharma AG, Pfizer, Allergan, Solvay (now Abbott), and Bayer. MP received consultancy fees and travel reimbursements from AstraZeneca, Novartis Pharma AG, Pfizer, Roche Diagnostics, and Takeda. CP received consultancy fees and travel reimbursement from Novartis Pharma AG. RS received consultancy fees and travel reimbursement from Novartis Pharma AG. US-E is a consultant for Alcon, Bayer, Novartis, and Pfizer.

The University of Vienna performs contract research as a clinical trial centre and Central Reading Centre.

\section{Acknowledgements}

We would like to acknowledge Laura Saunderson of Chameleon Communications International, who provided medical writing services with funding from Novartis Pharma AG, Basel, Switzerland. This encompassed preparation of the first draft, referencing, preparing tables and figures, and incorporating authors' revisions, all under the direction of the authors. At all stages, the authors have had full control over the content of this manuscript, for which they have given final approval and take full responsibility. Novartis Pharma AG enforces a 'no ghost-writing' policy. The expert panel meeting was sponsored by Novartis Pharma AG. As funding sponsors, they have had the opportunity to review the manuscript, but do not have authority to change any aspect of the manuscript. Funding for the expert panel meeting and medical writing services was provided by Novartis Pharma AG, Basel, Switzerland.

\section{References}

1 Aroca PR, Salvat M, Fernandez J, Mendez I. Risk factors for diffuse and focal macular edema. J Diabetes Compl 2004; 18: 211-215.

2 Ferris III FL, Patz A. Macular edema. A complication of diabetic retinopathy. Surv Ophthalmol 1984; 28(Suppl): 452-461.

3 Chen E, Looman M, Laouri M, Gallagher M, Van Nuys K, Lakdawalla D et al. Burden of illness of diabetic macular edema: literature review. Curr Med Res Opin 2010; 26: 1587-1597.

4 Mitchell P, Bandello F, Schmidt-Erfurth U, Lang GE, Massin P, Schlingemann RO et al. The RESTORE study: ranibizumab monotherapy or combined with laser versus laser monotherapy for diabetic macular edema. Ophthalmology 2011; 118: 615-625.

5 Massin P, Bandello F, Garweg JG, Hansen LL, Harding SP, Larsen $\mathrm{M}$ et al. Safety and efficacy of ranibizumab in diabetic macular edema (RESOLVE Study): a 12-month, 
randomized, controlled, double-masked, multicenter phase II study. Diabetes Care 2010; 33: 2399-2405.

6 Wild S, Roglic G, Green A, Sicree R, King H. Global prevalence of diabetes: estimates for the year 2000 and projections for 2030. Diabetes Care 2004; 27: 1047-1053.

7 American Academy of Ophthalmology Retina Panel. Preferred Practice Pattern guidelines: Diabetic Retinopathy. American Academy of Ophthalmology: San Francisco, CA, 2010.

8 The Royal College of Ophthalmologists. Guidelines for diabetic retinopathy. 2005. http://www.rcophth.ac.uk/ page.asp? section $=451 \&$ sectionTitle $=$ Clinical + Guidelines, 2011.

9 Early Treatment Diabetic Retinopathy Study Research Group. Photocoagulation for diabetic macular edema. Early Treatment Diabetic Retinopathy Study report number 1. Arch Ophthalmol 1985; 103: 1796-1806.

10 Elman MJ, Aiello LP, Beck RW, Bressler NM, Bressler SB, Edwards AR et al. Randomized trial evaluating ranibizumab plus prompt or deferred laser or triamcinolone plus prompt laser for diabetic macular edema. Ophthalmology 2010; 117: 1064-1077.

11 Elman MJ, Bressler NM, Qin H, Beck RW, Ferris III FL, Friedman SM et al. Expanded 2-year follow-up of ranibizumab plus prompt or deferred laser or triamcinolone plus prompt laser for diabetic macular edema. Ophthalmology 2011; 118: 609-614.

12 Funatsu H, Yamashita H, Noma H, Mimura T, Yamashita T, Hori S. Increased levels of vascular endothelial growth factor and interleukin- 6 in the aqueous humor of diabetics with macular edema. Am J Ophthalmol 2002; 133: 70-77.

13 Funatsu H, Yamashita H, Ikeda T, Mimura T, Eguchi S, Hori S. Vitreous levels of interleukin- 6 and vascular endothelial growth factor are related to diabetic macular edema. Ophthalmology 2003; 110: 1690-1696.

14 Bhagat N, Grigorian RA, Tutela A, Zarbin MA. Diabetic macular edema: pathogenesis and treatment. Surv Ophthalmol 2009; 54: 1-32.

15 Cunningham Jr ET, Adamis AP, Altaweel M, Aiello LP, Bressler NM, D'Amico DJ et al. A phase II randomized double-masked trial of pegaptanib, an anti-vascular endothelial growth factor aptamer, for diabetic macular edema. Ophthalmology 2005; 112: 1747-1757.

16 Michaelides M, Kaines A, Hamilton RD, Fraser-Bell S, Rajendram $\mathrm{R}$, Quhill $\mathrm{F}$ et al. A prospective randomized trial of intravitreal bevacizumab or laser therapy in the management of diabetic macular edema (BOLT study) 12-month data: report 2. Ophthalmology 2010; 117: 1078-1086.

17 Ahmadieh H, Ramezani A, Shoeibi N, Bijanzadeh B, Tabatabaei A, Azarmina M et al. Intravitreal bevacizumab with or without triamcinolone for refractory diabetic macular edema; a placebo-controlled, randomized clinical trial. Graefes Arch Clin Exp Ophthalmol 2008; 246: 483-489.

18 Diabetic Retinopathy Clinical Research Network. A randomized trial comparing intravitreal triamcinolone acetonide and focal/grid photocoagulation for diabetic macular edema. Ophthalmology 2008; 115: 1447-1449.

19 Soheilian M, Ramezani A, Obudi A, Bijanzadeh B, Salehipour M, Yaseri $\mathrm{M}$ et al. Randomized trial of intravitreal bevacizumab alone or combined with triamcinolone versus macular photocoagulation in diabetic macular edema. Ophthalmology 2009; 116: 1142-1150.

20 Do DV, Schmidt-Erfurth U, Gonzalez VH, Gordon CM, Tolentino M, Berliner AJ et al. The DA VINCI study: phase 2 primary results of VEGF Trap-Eye in patients with diabetic macular edema. Ophthalmology 2011; 118: 1819-1826.

21 Novartis Pharma AG. Summary of Product Characteristics 2011. Novartis Pharma AG: Basel, Switzerland, 2011. http:// emc.medicines.org.uk/emc/assets/c/html/ displaydoc.asp?documentid = 19409 (accessed 3 March 2011).

22 Nguyen QD, Shah SM, Heier JS, Do DV, Lim J, Boyer D et al. Primary end point (six months) results of the Ranibizumab for Edema of the mAcula in Diabetes (READ-2) Study. Ophthalmology 2009; 116: 2175-2181.

23 Nguyen QD, Shah SM, Khwaja AA, Channa R, Hatef E, Do DV et al. Two-year outcomes of the Ranibizumab for Edema of the mAcula in Diabetes (READ-2) study. Ophthalmology 2010; 117: 2146-2151.

24 Do DV, Nguyen QD, Khwaja AA, Channa R, Sepah YJ, Boyer DS et al. Aggressive anti-VEGF treatment is necessary to control diabetic macular edema in many patients: month 36 outcomes of the READ2 trial. The Association for Research in Vision and Ophthalmology 2011 Annual Meeting, 1-5 May 2011, p 5332.

25 Treatment techniques and clinical guidelines for photocoagulation of diabetic macular edema. Early treatment diabetic retinopathy study report number 2 . early treatment diabetic retinopathy study research group. Ophthalmology 1987; 94: 761-774.

26 Hansen LL. Visual acuity response to ranibizumab stratified by baseline characteristics in diabetic macular edema (RESOLVE Study). 20th Meeting of the European Association for the Study of Diabetic Eye Complications, 21-22 May 2010

27 Kannel WB, McGee DL. Diabetes and cardiovascular risk factors: the Framingham study. Circulation 1979; 59: 8-13.

28 Cheung N, Mitchell P, Wong TY. Diabetic retinopathy. Lancet 2010; 376: 124-136.

29 UK Prospective Diabetes Study (UKPDS) Group. Intensive blood-glucose control with sulphonylureas or insulin compared with conventional treatment and risk of complications in patients with type 2 diabetes (UKPDS 33). Lancet 1998; 352: 837-853.

30 UK Prospective Diabetes Study Group. Tight blood pressure control and risk of macrovascular and microvascular complications in type 2 diabetes: UKPDS 38. BMJ 1998; 317: 703-713.

31 Singh R, Abhiramamurthy V, Gupta V, Gupta A, Bhansali A. Effect of multifactorial intervention on diabetic macular edema. Diabetes Care 2006; 29: 463-464.

32 Ryan Jr EH, Han DP, Ramsay RC, Cantrill HL, Bennett SR, Dev $\mathrm{S}$ et al. Diabetic macular edema associated with glitazone use. Retina 2006; 26: 562-570.

33 Liazos E, Broadbent DM, Beare N, Kumar N. Spontaneous resolution of diabetic macular oedema after discontinuation of thiazolidenediones. Diabet Med 2008; 25: 860-862.

34 Ambrosius WT, Danis RP, Goff Jr DC, Greven CM, Gerstein HC, Cohen RM et al. Lack of association between thiazolidinediones and macular edema in type 2 diabetes: the ACCORD eye substudy. Arch Ophthalmol 2010; 128: 312-318.

35 Kuiper EJ, Van Nieuwenhoven FA, de Smet MD, Van Meurs JC, Tanck MW, Oliver $\mathrm{N}$ et al. The angio-fibrotic switch of VEGF and CTGF in proliferative diabetic retinopathy. PLoS One 2008; 3: e2675.

36 Oshima Y, Shima C, Wakabayashi T, Kusaka S, Shiraga F, Ohji $\mathrm{M}$ et al. Microincision vitrectomy surgery and intravitreal bevacizumab as a surgical adjunct to treat diabetic traction retinal detachment. Ophthalmology 2009; 116: 927-938. 